


\title{
PREMELTING OF ICE ADSORBED ON A ROCK SURFACE
}

\author{
V. Esteso \\ Multifunctional Optical Materials Group \\ Instituto de Ciencia de Materiales de Sevilla \\ (Consejo Superior de Investigaciones Científicas - Universidad de Sevilla), \\ Calle Américo Vespucio 49, 41092 Sevilla, Spain. \\ S. Carretero-Palacios*, \\ Multifunctional Optical Materials Group \\ Instituto de Ciencia de Materiales de Sevilla \\ (Consejo Superior de Investigaciones Científicas - Universidad de Sevilla), \\ Calle Américo Vespucio 49, 41092 Sevilla, Spain. \\ Departamento de Física de Materiales \\ Universidad Autónoma de Madrid \\ 28049 Madrid, Spain \\ sol.carretero@uam.es
}

\author{
L. G. MacDowell \\ Departamento de Química-Física \\ Facultad de Ciencias Químicas \\ Universidad Complutense de Madrid \\ 28040 Madrid, Spain.
}

\author{
J. Fiedler \\ Centre for Materials Science and Nanotechnology \\ Department of Physics \\ University of Oslo \\ P. O. Box 1048 Blindern, NO-0316 Oslo, Norway. \\ Physikalisches Institut \\ Albert-Ludwigs-Universität Freiburg \\ Hermann-Herder-Str. 3, 79104 Freiburg, Germany.
}

\section{F. Parsons}

School of Engineering and Information Technology Murdoch University

90 South St, Murdoch, WA 6150

Australia.

\section{F. Spallek}

Physikalisches Institut Albert-Ludwigs-Universität Freiburg Hermann-Herder-Str. 3, 79104 Freiburg, Germany.

\section{H. Míguez \\ Multifunctional Optical Materials Group Instituto de Ciencia de Materiales de Sevilla \\ (Consejo Superior de Investigaciones Científicas - Universidad de Sevilla), Calle Américo Vespucio 49, 41092 Sevilla, Spain.}

\section{Persson}

Centre for Materials Science and Nanotechnology

Department of Physics University of Oslo

P. O. Box 1048 Blindern, NO-0316 Oslo, Norway.

\section{Brevik}

Department of Energy and Process Engineering Norwegian University of Science and Technology NO-7491 Trondheim, Norway iver.h.brevik@ntnu.no

\section{S. Y. Buhmann}

Physikalisches Institut Albert-Ludwigs-Universität Freiburg Hermann-Herder-Str. 3, 79104 Freiburg, Germany.

\section{Boström*,}

Centre for Materials Science and Nanotechnology Department of Physics University of Oslo

P. O. Box 1048 Blindern, NO-0316 Oslo, Norway. Department of Energy and Process Engineering 


\begin{abstract}
Considering ice-premelting on a quartz rock surface (i.e. silica) we calculate the Lifshitz excess pressures in a four layer system with rock-ice-water-air. Our calculations give excess pressures across (1) ice layer, (2) water layer, and (3) ice-water interface for different ice and water layer thicknesses. We analyse equilibrium conditions where the different excess pressures take zero value, stabilised in part by repulsive Lifshitz interactions. In contrast to previous investigations which considered varying thickness of only one layer (ice or water), here we present theory allowing for simultaneous variation of both layer thicknesses. For a given total thickness of ice and water, this allows multiple alternative equilibrium solutions. Consequently the final state of a system will depend on initial conditions and may explain variation in experimental measurements of the thicknesses of water and ice layers.
\end{abstract}

\title{
1 Introduction
}

In the physical process of ice melting near or at the triple point of water, a nano-sized film of water has been predicted to occur at the surface of ice. It can be stabilised by repulsive Casimir (Lifshitz) forces, [1, 2, 3] and under variable conditions (e.g. different temperatures, presence of salt ions and impurities, [3, 4, 5, 6, 7] diverse geometries, [7, 8] etc) thicker or thinner films can occur. Beside effects arising from impurity charges, [4, 5, 9] diverse strategies have been proposed that could also lead to the formation of thicker liquid water films. One way is the addition of a thin hydrocarbon coating on ice; this may substantially increase the water sheet thickness on the surface of melting ice. [10] In a similar way, the premelting water layer thickness for ice in cavities can be enhanced by the presence of a gas layer between ice and the pore surface. [11] Additional effects can potentially arise from ion-specific hydration and ion-specific dispersion forces between ions and interfaces, [12, 13, 14, 15, 16].

The thickness of liquid water layers on ice surfaces has strong environmental implications: it needs to be at least micron sized to be relevant as a habitat for any known lifeforms, and its formation may potentially influence the charging of thunderclouds [17, 18, 19] and frost heave. [6, 20] Moreover, suggestions have been made about the existence of liquid water due to ice premelting on icy moons and planets. [21, 22, 23, 24, 25] Along these lines, Casimir (Lifshitz) forces have further been proposed to be able to cause ice formation on some gas hydrate surfaces in water. [26] Such ice films, if present, could induce a size-dependent buoyancy for $\mathrm{CO}_{2}$ gas hydrate clusters, for instance, in the underlying oceans on the moons Enceladus and Europa, potentially influencing any harboured life. [26]

However, the problem of ice premelting and particularly the characterisation of a reliable equilibrium film thickness has remained a controversial topic for many years. [2, 21, 22, 27, 7] Experimental results referring to the thickness of the equilibrium premelting layer at the ice-vapour interface performed since the 1980's differed by orders of magnitude. [28] Fortunately, in recent times results from widely different experimental techniques, that had been in serious conflict previously, seem to be converging towards values that appear to be in reasonable agreement with computer simulations. [29] Based on these studies, [30, 31, 9] the equilibrium ice surface exhibits a thin premelting layer of barely $1 \mathrm{~nm}$ a few Kelvin away from the triple point, but can considerably increase to tens of nanometers some tenths of Kelvin away from the triple point. The central question as to whether the film thickness diverges or remains finite at the triple point seems however still difficult to elucidate because of the large effect of salt or charge on the ice surface [32, 5], or of small amounts of impurities. [4, 9]

In this context, Lifshitz's theory of intermolecular forces — London dispersion forces - seems the most reliable educated guess, and within this framework, the answer is essentially dictated by the difference between the refraction indexes of water and ice. Specifically, this difference being positive in some frequency region and negative in others, yields a crossing of the dielectric functions and shows the potential of a water premelting film to remain finite at the triple point. [1]

Here, we present a theoretical study of the premelting behaviour of ice adsorbed on a rock surface. First we provide some background to the field of intermolecular interactions both in the general case and for ice-water systems. The theory for Lifshitz excess pressures in a 4-layer system is then presented together with some numerical examples. Previous theories for Casimir (Lifshitz) forces considered only the option to have a single film thickness that can vary at a time. A theory that enables ice and water layer thicknesses to vary independently is derived here. As a vital test the new theory does, in the appropriate limit, recover the classical premelting layer on ice surfaces predicted by Elbaum and Schick. [1] Our study reveals a more complex and interesting picture when the complete theory is used. If the films of ice as well as of water are thin, energy considerations lead to one or more possible equilibria depending on the initial conditions. 


\section{Dispersion force for 4-layer systems}

Dispersion forces, in general, are interactions between polarisable objects, such as atoms and molecules, described by their polarisabilities, and dielectric objects via their corresponding permittivities. Thus, dispersion forces include the London-van-der-Waals interaction between two polarisable particles; the Casimir-Polder interaction between a polarisable particle and a dielectric object; and the Casimir effect as the interaction between two dielectric objects. Its origin is the ground-state fluctuations of the electromagnetic fields [33]. Alternative accounts derive dispersion forces from position dependent ground-state energies (zero-point energies) of the coupled field-matter system [34, 35]. The physical mechanism behind these forces can be understood by the spontaneous creation of polarisations inside the interacting particles caused by the field fluctuations. The resulting polarisations can then interact with each other and yields the macroscopic force. Modern theories [36, 37] apply Green's function techniques to separate the interactions into the particle's properties and the environmental properties (including the geometry of the system). The latter are represented by the Green's function, which is the fundamental solution of the Helmholtz equation, and thus describe the interaction in terms of an exchange of virtual photons. Consequently, these methods express dispersion interactions via classical optical properties, such as reflection and transmission at interfaces. In the following, we will use these terms to derive the dispersion interactions in the four-layer system. It should be stressed that ultimately, all dispersion forces involving macroscopic objects can be understood as sums over microscopic many-atom Van der Waals forces between the respective constituent atoms.

\subsection{A brief history of intermolecular dispersion forces}

Before we set out to study melting of ice absorbed on a surface, it may be worthwhile to revisit some important developments in the history of intermolecular forces in a wider context. A historical, almost 4000 years old, set of "experiments" were discussed by Tabor [38] when he presented translation and discussion of a clay tablet [39] that according to Tabor dates back to the time of Hammurabi (a Babylonian ruler from an area which is now in Iraq [40]) around 18th century before Christ. [38] The priests were studying oil on water spreading (pouring water in oil and oil in water) for various religious, mystical, or transcendental reasons. There were, according to Tabor, various scientifically interesting results in this early publication such as spreading and retraction, the formation of droplets and globules, and interference colours. [38] 1

Thomas Young discussed attractive and repulsive forces in liquids in his article "An essay on the cohesion of fluids" in 1805. [41] As mentioned in a review of Dash, [42] it seems that Michael Faraday was the first to observe that a pressure acting on an ice sheet can result in a melting of the sheet. However, as discussed by Derjaguin, Abrikossova, and Lifshitz in their review paper from 1956 [43] the correct idea about the nature of molecular forces, relating intermolecular forces to radiation processes, was first put forward by P. N. Lebedev in 1894 [44] (predating quantum theory). For small interparticle distances, the van der Waals interaction between two atoms in the non-retarded limit was explained by London in terms of frequency-dependent responses to the electromagnetic quantum fluctuations in the two interacting polarisable atoms. [45] The subsequent theory derived by Casimir and Polder led to a proper understanding of dispersion forces between atoms, [35] between an atom and a metal surface, [35] and between two perfect metal surfaces, [34] and related the results to the zero-point energy of the vacuum. Lifshitz and co-workers derived a more general theory for Casimir forces between surfaces, [46, 47] that determines the interaction in terms of dielectric functions of the interacting objects and of intervening media. Dielectric functions are related to refractive index and adsorption spectra of the specific media, thus proving the relationship between forces and radiation processes proposed by Lebedev. [44, 43] The derivation of the Lifshitz theory was simplified, [48, 49] and made useful for multilayered systems [49, 50, 51, 52, 53] as well as extended to include magnetic effects [54] by Ninham, Parsegian and co-workers. [55]

As was clear to the original authors [47], these Lifshitz forces (also called Casimir forces) are often attractive but can also be repulsive. The force can be repulsive if the dielectric function of the intervening medium lies between that of the two planar interacting surfaces for some frequency range. Beyond this possibility creating repulsive dispersion forces, anisotropic particles or magnetically responsive particles can feel a repulsive force as well. [56] This repulsive frequency range then has to dominate the influence from an eventual other attractive frequency range in order to make the force repulsive. [57, 51, 53, 58, 59, 60, 14, 37, 61, 62, 63, 64, 65] The transition distance from attractive to repulsive Lifshitz force occurs when the attractive and repulsive frequency contributions exactly cancels. [53] Pioneering studies of liquid helium films on different substrates have in the past clearly shown the effect of repulsive Lifshitz forces. [66, 51, 67, 68]

\footnotetext{
${ }^{1}$ As a note we would like to mention that George Smith found hundreds of fragmentary clay tablets from the library of Nineveh in Assyria on the Tigris river. These described among other things an older version of the biblical story of the flooding in the Gilgamesh epic and more. [40]
} 


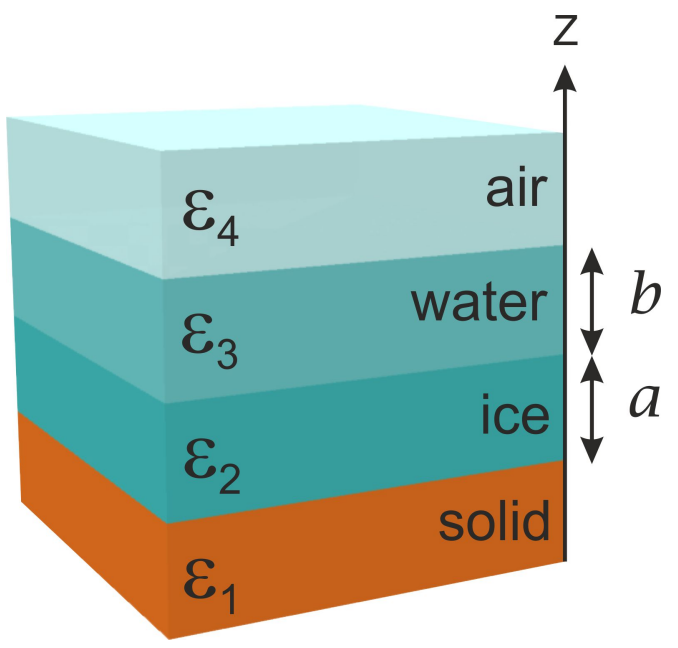

Figure 1: Schematic figure of the four layer system: solid layer (1) of infinite thickness and dielectric function $\varepsilon_{1}$ (quartz), followed by an ice layer (2) with dielectric function $\varepsilon_{2}$ of thickness $a$, enclosed by a liquid water layer (3) with dielectric function $\varepsilon_{3}$ and thickness $b$ continued by an infinite air layer (4) with dielectric function $\varepsilon_{4}=1$.

A great step towards the unification of dispersion forces and generalisation to arbitrary geometries occurred with the quantisation of electromagnetic fields in dispersive and absorbing media . 69, 36]

Hauxwell and Ottewill [70] performed measurements related to spreading of alkanes on water due to dispersion forces ${ }^{2}$ Reasonable agreement between experimental results and theory was found already in the 1970s. [57, 51, 53] Recent force measurements have also seen repulsive Lifshitz forces, [71, 72, 73, 74] and these have been lately demonstrated through spectroscopic techniques. [75, 65]

In a general context, the books by Bordag, Klimchitskaya, Mohideen, and Mostepanenko, [76] Milton, [77], Parsegian [78], Ninham and Lo Nostro [79], Buhmann, [37, 61] Simpson and Leonhardt, [80] and Sernelius, [62] deserve special attention.

Opening up the subject that concerns our studies, the contribution from dispersion free energy to surface tension of water has been estimated to be around $0.02 \mathrm{~N} \mathrm{~m}^{-1}$ while the measured surface tension at room temperature is around $0.072 \mathrm{~N} \mathrm{~m}^{-1}$. [81] Of course, interactions of water or ice with a substrate, or among ice and water have also a significant structural component related to packing effects and hydrogen bonding. However, these interactions are invariably short range in the sense that they decay exponentially fast, with a correlation length of the order of a few molecular diameters. [82, 83, 84, 85] For small distances, the structural forces can be quite large, and can very much influence the thin film equilibrium state. However, for films several nanometers thick, Van der Waals interactions largely dominate the overall behaviour. Casimir (Lifshitz) theory is then an essential tool, because the quantum electrodynamical nature of the interactions at long range precludes a reliable estimation by point-charge non-polarisable models that are most often employed in molecular dynamics simulations. [29, 86, 87, 88]

At this point, we would like to mention that the solubility of dissolved gases (both as gas molecules and bubbles) in water [14, 89, 90] also influences intermolecular forces and melting of ice. For instance, dissolved $\mathrm{CO}_{2}$ gas molecules in water influence the formation of ice layers on $\mathrm{CO}_{2}$ gas hydrates in water at the quadruple point. [26]

\subsection{4-layer system definition}

To analyse ice-premelting on a rock surface we calculate the Casimir (Lifshitz) excess pressure in a four layer scheme as the one depicted in Fig. 11, which serves as a general model for solid-ice-water-air arrangements, corresponding to regions $i=1,2,3$ and 4 , respectively. In the scheme, the origin of the coordinate system along the $z$-axis, i.e. $z=$ 0 , is set at the interface between the ice layer of thickness $a$ and the water layer of thickness $b$. We assume the solid and vapour layer thicknesses to be large compared to $a$ and $b$, which allows us to set the corresponding thicknesses to infinity.

\footnotetext{
${ }^{2}$ Prof. B. W. Ninham pointed out to us that in the experiments by Hauxwell and Ottewill the control of vapour pressure might not have been done in a sufficiently accurate way.
} 
From the outset, we consider that our system is in thermal and chemical equilibrium, i.e., all 4 phases have the same temperature and chemical potential. Full thermodynamic equilibrium further requires that the perpendicular pressure to the interface be the same in all four layers, and this additional constraint sets equilibrium values for the free parameters $a$ and $b$ corresponding to the layer thickness of medium 2 (ice) and 3 (water). Of course, at the triple point the bulk ice, water and water vapour phases meet simultaneously the condition of equality of temperature, chemical potential and pressure. Therefore, a trivial solution corresponds to $a \rightarrow \infty$ and $b \rightarrow \infty$, such that the finite ice and water layers become the actual bulk phases. However, the equality of pressure for the four phases can also be achieved for finite $a$ and $b$, due to the interplay of intermolecular forces across the four media.

In the next section we formulate how Casimir (Lifshitz) forces between adjacent media across an area $A$, effectively result in excess pressures between the media. Therefore, at the triple point, where bulk pressures are equal, the excess pressures resulting from Casimir (Lifshitz) forces can set additional equilibrium conditions for finite $a$ and $b$. In section 3.3 we will then discuss how the results for the Casimir (Lifshitz) 'radiative pressure' can be extended to the case where bulk pressures are different (i.e., away from the triple point).

\subsection{Theory of dispersion forces in planar layered media}

\subsubsection{Energy approach}

In order to clarify the discussion we stress that the dispersion interaction energy in a planar system is simply due to the change in energy of the electromagnetic normal modes of the system with separation. The electromagnetic energy per unit surface is

$$
E=\sum_{j} \hbar \omega_{j}\left[n\left(\omega_{j}\right)+\frac{1}{2}\right], \quad n(\omega)=1 /\left[e^{\beta \hbar \omega}-1\right],
$$

with $\beta=1 / k_{\mathrm{B}} T$, and $\omega_{j}$ are the frequencies of the electromagnetic normal modes. At zero temperature, $n\left(\omega_{j}\right)=0$ and only the zero-point energy appears. At finite temperature, the other terms also contribute. The surface force per unit area (i.e. pressure) may be calculated as the derivative $F=-\mathrm{d} \mathcal{F} / \mathrm{d} a$, where $\mathcal{F}=E-T S$ is the Helmholtz free energy with $S$ being the entropy.

In the current work we present how to treat systems where more than one thickness is allowed to vary independently. The electromagnetic modes are found by solving Maxwell's equations under standard electromagnetic boundary conditions. The obtained eigenmodes are massless bosons, each with an energy corresponding to a quantum oscillator. These energies can be shown, see for instance Boström and Sernelius, [91] to be equivalent to the expression given much earlier by Lifshitz and co-workers, [47] and in the 1970s by Ninham and co-workers. [49, 48] The analysis depends on the dielectric function taken as a complex function with real and imaginary components. But these components, and their values at real frequencies and at imaginary frequencies are not independent. They are related through Kramers-Kronig transformations, which express the causality of physical processes such as polarisation in continuous matter. In particular, the Kramers-Kronig relation most relevant to Lifshitz theory, linking the dielectric function at real and imaginary frequencies, is

$$
\varepsilon(\omega)=1+\frac{2}{\pi} \int_{0}^{\infty} \frac{x \varepsilon^{\prime \prime}(x)}{x^{2}+\omega^{2}} \mathrm{~d} x,
$$

where $\varepsilon=\varepsilon^{\prime}+\varepsilon^{\prime \prime}$. Notice that whereas $\varepsilon(\omega)$ is a complex function, the corresponding result evaluated at imaginary frequencies is purely real, as evidenced by the Kramers-Kronig relation here.

The Lifshitz energy can be calculated in different mathematically identical ways: as an integration over real frequencies over quantities essentially related to optical absorption or in a numerically much easier way as a summation over imaginary frequencies. [91] In the following subsections we only give the final results for a 4-layer system. [92, 93] The novel aspect of our work is that we allow an interface to be influenced by independent forces from either side. The overall force is simply the difference of these two forces. To give an explicit example, if an outward pointing force is acting across the ice layer and in same way another outward pointing force is acting on the water layer the different forces will have opposite directions (signs) when they act on the intermediate interface. This will be clarified mathematically in the next subsection.

For dispersion force calculations, parameterised dielectric functions of all materials involved need to be known, corresponding to the optical response via the Kramers-Kronig relation in Eq. 22, i.e. $\varepsilon_{i}\left(\xi_{m}\right)$, with the Matsubara frequencies [61] $\xi_{m}=\left(2 \pi k_{\mathrm{B}} T / \hbar\right) m$, where $k_{\mathrm{B}}$ is Boltzmann's constant, $\hbar$ is the reduced Planck's constant, and $m=$ $0,1,2, \ldots$ We use silica as a typical quartz rock material, whose dielectric function $\varepsilon_{1}\left(\xi_{m}\right)$ is taken from van Zwol and Palasantzas. [59] The parameterised dielectric functions for ice, $\varepsilon_{2}\left(\xi_{m}\right)$, and water, $\varepsilon_{3}\left(\xi_{m}\right)$, are taken from Ref. 1] in 


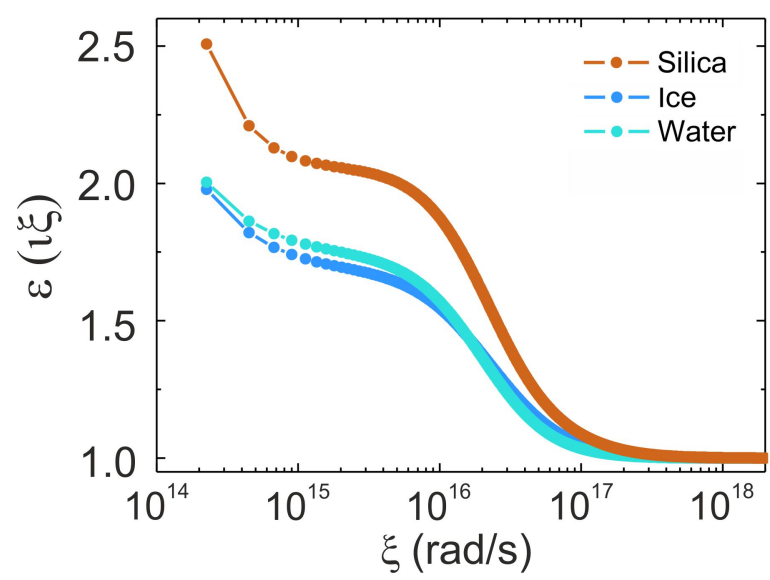

Figure 2: Dielectric function evaluated at the imaginary frequencies for water [1] (light blue), ice [1] (blue) and silica [59] (brown). Circles stand for the discrete imaginary frequencies contributing to the Casimir (Lifshitz) interaction at $T=273.16 \mathrm{~K}$.

which the system is assumed to be close to the triple point of water $(T=273.16 \mathrm{~K}$ and a partial vapour pressure of $611.73 \mathrm{~Pa}$ ). Finally, vapour is set to be $\varepsilon_{4}\left(\xi_{m}\right)=1$. Figure 2 shows $\varepsilon_{i}\left(\xi_{m}\right)$ of all materials considered here.

\subsubsection{Stress tensor approach}

The Casimir (Lifshitz) force on a unit of surface area $(\mathrm{d} A)$ can also be obtained via a surface integral over a closed mathematical surface that encloses the physical surface, [37] redJF: Is the surface really closed for planar systems?

$$
\mathbf{F}=\oint \mathrm{d} \mathbf{A} \cdot\langle\hat{\mathbf{T}}\rangle
$$

with the quantum mechanical expectation value of the electromagnetic stress tensor $\langle\hat{\mathbf{T}}\rangle$, which reads [94]

$$
\hat{\mathbf{T}}=\hat{\mathbf{D}} \hat{\mathbf{E}}+\hat{\mathbf{H}} \hat{\mathbf{B}}-\frac{1}{2}(\hat{\mathbf{D}} \cdot \hat{\mathbf{E}}+\hat{\mathbf{H}} \cdot \hat{\mathbf{B}}) \mathbb{I} .
$$

Here the three dimensional unit matrix is $\mathbb{I}=\operatorname{diag}(1,1,1)$. The fields are the standard electromagnetic fields: electric field $(\hat{\mathbf{E}})$, displacement field $(\hat{\mathbf{D}})$, magnetic induction field $(\hat{\mathbf{B}})$ and magnetic field $(\hat{\mathbf{H}})$. For this work we adopt a planar geometry with the surface normal pointing towards the $z$-direction, and assume a unit surface area so that the surface force $F$ becomes the same as the surface force per unit area (pressure). It is perpendicular to the surface, and can be written

$$
F=\left.\left\langle\hat{T}_{z z}\right\rangle\right|_{z=0+}-\left.\left\langle\hat{T}_{z z}\right\rangle\right|_{z=0-}
$$

(note that the first term in this equation refers to the outer surface $z=0+$; if the $z z$ component of the stress tensor is positive, it means that the force acts in the positive $z$ direction). The force acting on the interface between layers 2 and 3 (i.e., ice-water) can thus be written as the difference of the stresses in layers 2 and 3.

Moreover, the surface excess pressure on the "bottom" of the water layer has to be equal and negative to the surface excess pressure on its "top",

$$
F_{3, \text { bottom }}=-F_{3, \text { top }}
$$

and so, the pressure on the ice-water layer becomes

$$
F_{2-3}=F_{2, \text { top }}-F_{3, \text { top }},
$$

where each component can be estimated via a three-layer Casimir force with the respective reflection coefficient taking into account the multiple reflections [37] at the additional interface. We note that Eq. (7) goes beyond the pioneering studies on multilayered Lifshitz forces by Ninham and co-workers. [49, 50, 51, 52, 53] The work of Parsegian and Ninham [52] for a system with interacting multilayers with arbitrary number of layers presented the Lifshitz energy as an interaction energy $G(l)$ with respect to the thickness $l$ of one of the layers, with the remaining layers describing coatings of fixed thickness. In this Parsegian-Ninham model, $G(l) \rightarrow 0$ as $l \rightarrow \infty$, regardless of the thickness of other layers. Our purpose here is to have layers 2 and 3 on exactly the same footing such that both may vary in thickness (see Section 2.5, allowing $G(l)$ to be non-zero as $l \rightarrow \infty$. 


\subsubsection{Surface excess pressure on the top water surface}

We explore the Lifshitz theory in layered nonmagnetic media. This can be derived in different equivalent ways: via the energy of the electromagnetic modes (see Sec. 2.3.1) or via the stress tensor approach (see Sec. 2.3.2) The surface excess pressure acting on top of the water layer is expressed as [92, 93]

$$
F_{3, \text { top }}=-\frac{k_{\mathrm{B}} T}{\pi} \sum_{m=0}^{\infty} \int_{0}^{\infty} \mathrm{d} k^{\|} k^{\|} \kappa_{3}^{\perp} \sum_{\sigma=\mathrm{TE}}^{\mathrm{TM}} \frac{1}{D_{\sigma 3}} .
$$

Here $\mathbf{k}^{\|}$is the component of the wave vector $\mathbf{k}=(2 \pi / \lambda) \mathbf{e}_{k}$ parallel to the surface, and $\kappa_{i}^{\perp}=\sqrt{k^{\|^{2}}+\varepsilon_{i} \xi_{m}^{2} / c^{2}}$ (with $i=1,2,3,4$ and $\xi_{m}$ is the Matsubara frequency defined above) is a real quantity. As stated above, this same pressure can equivalently be given as an integral over real frequencies of photon-pressure contributions from various light rays, our reformulation in terms of imaginary frequencies is purely for mathematical convenience. In the language of geometric optics, such light rays are subjected to multiple reflections from the dielectric boundaries. In the context of Lifshitz theory, we interpret this as multiple reflections of the virtual photons (with imaginary frequencies $\xi$ ) which drive dispersion interactions. One then needs the expressions for the Fresnel reflection coefficients, which we shall call $r_{\sigma}^{i j}$, in which a ray in medium $i$ is assumed to be reflected from medium $j$. The photon polarisations are $\sigma=\mathrm{TE}$ (transverse electric, also called $s$-polarised), and $\sigma=\mathrm{TM}$ (transverse magnetic, or $p$-polarised). The primed sum in the expression above denotes that the first term $(m=0)$ has to be weighted by $1 / 2$.

In order to take into account the multiple reflections between the interface, we need the following expression for the mode structure (for details see the References [92, 93]),

$$
\frac{1}{D_{\sigma 3}}=\frac{\tilde{r}_{\sigma}^{32} r_{\sigma}^{34} \mathrm{e}^{-2 \kappa_{3}^{\perp} b}}{1-\tilde{r}_{\sigma}^{32} r_{\sigma}^{34} \mathrm{e}^{-2 \kappa_{3}^{\perp} b}} .
$$

To include the additional interface (here at layers 1 and 2) one needs also the generalised reflection coefficient

$$
\tilde{r}_{\sigma}^{32}=\frac{r_{\sigma}^{32}+r_{\sigma}^{21} \mathrm{e}^{-2 \kappa_{2}^{\perp} a}}{1+r_{\sigma}^{32} r_{\sigma}^{21} \mathrm{e}^{-2 \kappa_{2}^{\perp} a}} .
$$

The explicit expressions for the well-known Fresnel reflection coefficients between surfaces $i$ and $j$ are for the two polarisations

$$
\begin{gathered}
r_{\mathrm{TE}}^{i j}=\frac{\kappa_{i}^{\perp}-\kappa_{j}^{\perp}}{\kappa_{i}^{\perp}+\kappa_{j}^{\perp}}, \\
r_{\mathrm{TM}}^{i j}=\frac{\varepsilon_{j} \kappa_{i}^{\perp}-\varepsilon_{i} \kappa_{j}^{\perp}}{\varepsilon_{j} \kappa_{i}^{\perp}+\varepsilon_{i} \kappa_{j}^{\perp}} .
\end{gathered}
$$

\subsubsection{Surface excess pressure on the top ice surface}

Analogously, one finds the surface excess pressure acting on top of the ice surface [92, 93]

$$
F_{2, \text { top }}=-\frac{k_{\mathrm{B}} T}{\pi} \sum_{m=0}^{\infty}, \int_{0}^{\infty} \mathrm{d} k^{\|} k^{\|} \kappa_{2}^{\perp} \sum_{\sigma=\mathrm{TE}}^{\mathrm{TM}} \frac{1}{D_{\sigma 2}},
$$

where

$$
\frac{1}{D_{\sigma 2}}=\frac{r_{\sigma}^{21} \tilde{r}_{\sigma}^{23} \mathrm{e}^{-2 \kappa_{2}^{\perp} a}}{1-r_{\sigma}^{21} \tilde{r}_{\sigma}^{23} \mathrm{e}^{-2 \kappa_{2}^{\perp} a}} .
$$

In this case, the generalised reflection coefficient for the reflection at the interface between layer 2 and 3 is required

$$
\tilde{r}_{\sigma}^{23}=\frac{r_{\sigma}^{23}+r_{\sigma}^{34} \mathrm{e}^{-2 \kappa \frac{1}{3} b}}{1+r_{\sigma}^{23} r_{\sigma}^{34} \mathrm{e}^{-2 \kappa \frac{1}{3} b}} .
$$




\subsection{Screening of the $m=0$ term in the Lifshitz pressure}

Surface charges and salt ions influence ionic double layer interactions as well as via mobile ions screening the zero frequency part $(m=0)$ of the Lifshitz interaction. Especially, at high salt concentrations ionic double layer interactions are screened out [4, 5] but so is the zero frequency contribution to the Lifshitz pressure. In order to take into account how the presence of salt in the liquid water phase influences the zero frequency part of the transverse magnetic contribution to the Lifshitz excess pressure one replaces $\kappa_{3}^{\perp}(m=0) \rightarrow \sqrt{k^{2}+\kappa_{\mathrm{D}}^{2}}$ where the magnitude of the Debye vector takes the form $\kappa_{\mathrm{D}}=e \sqrt{8 \pi I /\left[\varepsilon_{3}(m=0) k_{\mathrm{B}} T\right]}$, as has been analysed by Ninham and Yaminsky. [55, 12] Here $I$ is the ionic strength of the electrolyte (the salt concentration, in the case of 1:1 electrolytes). The results presented in the result section assumes absence of salt ions and no surface charges. However, all curves can easily be modified by including a salt concentration dependent correction factor $\Delta F(I)=\left[F_{m=0}(I)-F_{m=0}(I=0)\right]$. In situations where the $m=0$ term plays a dominant role it is vital to consider the important work by Ninham and Yaminsky, [12] as well as pondering the relevance of the more recent work from Maia Neto and co-workers. [95] The fundamental differences related to screening across electrolytes observed in some of the publications that we have discussed [12, 95] will likely be the topic of future investigations. As already mentioned, we do not consider cases with Lifshitz forces acting across such intervening electrolytes.

\subsection{Thermodynamic formulation}

In the Lifshitz theory, the Casimir (Lifshitz) forces are measured as an excess over those corresponding to infinite macroscopic bodies, and they arise essentially due the condition of standing electromagnetic waves at a given finite distance between the bodies, i.e., they do not take into account the external bulk pressure within the bodies, which must be added ad hoc.

A full thermodynamic free energy functional can be considered which incorporates both effects simultaneously and leads naturally to an equilibrium condition as a function of imposed pressure and temperature. In our system, macroscopic layer 1 is actually an inert solid phase, which we can assume has constant volume, $V_{1}$. Layers 2,3 and 4 (of corresponding volumes $V_{2}, V_{3}$, and $V_{4}$ ), on the other hand, correspond to ice, water, and water vapour, respectively, and grow one at the expense of the other depending on the overall pressure in each phase. Let the Lifshitz free energy due to confinement be given by the function $G(a, b)$, and allow the bodies to have a bulk thermodynamic pressure $p_{i}(T, \mu)$. Then, the full free energy is given as: 96

$$
\Omega=-p_{2} V_{2}-p_{3} V_{3}-p_{4} V_{4}+A G(a, b) .
$$

Accepting that we can control the overall constant chemical potential by tuning the pressure of the vapour phase, $p_{4}=p(T, \mu)$, and that the total size of the system (ignoring the inert phase) is constant, such that $A \cdot\left(a+b+L_{4}\right)=V$ (with $L_{4}$ the thickness of the vapour layer, and $V$ constant total volume), we can write

$$
\frac{\Delta \Omega}{A}=-\left(p_{2}-p\right) a-\left(p_{3}-p\right) b+G(a, b) .
$$

At fixed temperature and chemical potential, all bulk pressures are constant, and the equilibrium values adopted by the ice and liquid layers are given as

$$
\left\{\begin{array}{l}
\frac{\partial}{\partial a}\left(\frac{\Delta \Omega}{A}\right)=0 \\
\frac{\partial}{\partial b}\left(\frac{\Delta \Omega}{A}\right)=0
\end{array}\right.
$$

This leads to the condition

$$
\left\{\begin{array}{l}
-\left(p_{2}-p\right)-F_{2, \text { top }}=0 \\
-\left(p_{3}-p\right)-F_{3, \text { top }}=0
\end{array}\right. \text {. }
$$

In the most general case this is a complicated equation for the equilibrium layer thickness as a function of the vapour pressure and temperature. For points along the sublimation line, $p=p_{2}$, and the second line of this system of equations then yields the equilibrium layer thickness of water on ice as a function of temperature. Here, we further simplify this complex problem and consider the triple point condition, where $p_{2}=p_{3}=p_{4}$, and we are left with the condition $F_{2-3}=F_{2 \text {,top }}-F_{3, \text { top }}=0$ for equilibrium of ice and water layers at the quartz/vapour interface in the triple point.

The Casimir (Lifshitz) dispersion energy for multilayered materials has been known for a long time. [49, 50, 51, 52, 53] However, in the usual formulation, one has been concerned with the free energy between materials 1 and 4 , coated by a third material 3, as a function of the materials thickness 2. Particularly, one has, in conventional applications: [49]

$$
G(a ; b)=\frac{k_{\mathrm{B}} T}{2 \pi} \sum_{m=0}^{\infty}, \int_{0}^{\infty} \mathrm{d} k^{\|} k^{\|} \ln D(a ; b)
$$


where $D(a ; b)=0$ is the dispersion relation for the surface electromagnetic modes in the system, and the function $D(a ; b)=D_{\mathrm{TM}}(a ; b) D_{\mathrm{TE}}(a ; b)$, with

$$
D_{\sigma}(a ; b)=1-r_{\sigma}^{21} \tilde{r}_{\sigma}^{23} e^{-2 \kappa_{2}^{\perp} a}
$$

This expression is not suitable for our purposes, as it is not symmetrical with respect to the interchange of media 2 and 3. The reason is that, as written here, it is meant to describe a coated substrate with layer 3 fixed at thickness $b$. As a result, the function $D_{\sigma}(a ; b)$ has been normalised to enforce that $G(a ; b) \rightarrow 0$ as $a \rightarrow \infty$ at constant and finite $b$. A fully equivalent, but more general form $D_{\sigma}(a, b)$ with the variables $a$ and $b$ on the same footing, can be constructed by multiplying $D_{\sigma}(a ; b)$ above by the denominator of Eq. $\left[15\right.$, which we write here as $1-r_{\sigma}^{32} r_{\sigma}^{34} e^{-2 \kappa_{3} b}$, using the fact that $r_{\sigma}^{32}=-r_{\sigma}^{23}$. This merely changes the normalisation of the free energy, which is now enforced to vanish for both $a$ and $b \rightarrow \infty$ simultaneously. The symmetrised free energy reads:

$$
G(a, b)=\frac{k_{B} T}{2 \pi} \sum_{m=0}^{\infty} \int_{0}^{\infty} \mathrm{d} k^{\|} k^{\|} \ln D(a, b)
$$

with

$$
D_{\sigma}(a, b)=1-r_{\sigma}^{21} r_{\sigma}^{23} e^{-2 \kappa_{2}^{\perp} a}-r_{\sigma}^{32} r_{\sigma}^{34} e^{-2 \kappa_{3}^{\perp} b}-r_{\sigma}^{21} r_{\sigma}^{34} e^{-2 \kappa_{2}^{\perp} a-2 \kappa_{3}^{\perp} b}
$$

This result may be obtained rigorously from the full dispersion relation of the system, and is now explicitly symmetrical with respect to the interchange of materials 2 and 3 , as expected (one can verify that the ratio $D_{\sigma}(a, b) / D_{\sigma}(a \rightarrow \infty, b)$ with $D_{\sigma}(a, b)$ from Eq. 23 recovers Eq. 21) exactly). Also by taking partial derivatives of Eq. (22) with respect to either $a$ or $b$ one can readily verify that Eq. (13) and Eq. (8) for $F_{2, \text { top }}$ and $F_{3, \text { top }}$ are recovered, respectively. The above expression has also a very suggestive form, since, for small $r_{\sigma}^{i j}$ or large $a$ and $b$ values, one readily finds that the free energy can be expressed as:

$$
G(a, b) \approx G_{123}(a)+G_{234}(b)+G_{1234}(a+b)
$$

where $G_{123}(a)$ is the free energy between media 1 and 3, filled with medium 2 of thickness $a$, while $G_{1234}(a+b)$ accounts for the interaction between media 1 and 4 across the compound layer formed of 2 and 3 . This is the expected form for purely additive dispersive forces calculated $a$ la Hamaker. Obviously, for $a \rightarrow \infty$, one finds $G(a, b)=G_{234}(b)$, so that the free energy is essentially dictated by the thickness of media 3 as sandwiched between media 2 and 4 . For the conventional application with $b$ fixed, the symmetrised result just switches the zero of energies, and is therefore removed so that $G(a ; b)=G(a, b)-G_{234}(b)$ when variations of the coating thickness $b$ are not a concern.

\section{Results}

\subsection{Premelting in the Elbaum-Schick limit}

Figure 3 shows the surface pressure, at the triple point, on top of the water layer as a function of the water layer thickness $b$, in the 4-layer system when $a \rightarrow \infty$ (i.e. the Casimir interaction between quartz and vapour is negligible due to the large thickness of the ice layer). This configuration is equivalent to the one proposed by Elbaum and Schick [1] (ice-water-vapour) as $a$ tends to the limit of being infinite. Positive values of the pressure indicates a repulsive interaction between the semi-infinite ice and vapour through the water layer, while negative values indicate an attractive interaction. For $b=3.6 \mathrm{~nm}$, pressure becomes zero, which is in agreement with the minimum in energy found by Elbaum and Schick. [1] This result shows that growth in the water layer is driven by repulsive Casimir (Lifshitz) interactions when $b<3.6 \mathrm{~nm}$, whereas the water layer become thinner when $b>3.6 \mathrm{~nm}$, due to attractive interactions.

\subsection{Ice formation at the solid-water interface}

Boström et al. [97] explored, in the spirit of Elbaum and Schick, [98] if Lifshitz forces at the triple point of water could cause ice formation at water-solid interfaces. It was suggested that Lifshitz forces can promote thin films of ice on some water-silica interfaces, [97] but not on water surfaces. [98] In the appropriate limits the result should come out again in our more general theory. As expected, in Figure 4 it is seen that a $5.4 \mathrm{~nm}$ thick film of ice leads to an equilibrium situation with zero pressure when the water layer is very thick, i.e. when $b \rightarrow \infty$.

\subsection{Premelting and ice growth in the general case}

In order to gain insight into premelting at the triple point, we extend the Casimir (Lifshitz) pressure analysis to finite values for both $a$ and $b$ at $T=273.16 \mathrm{~K}$. The Casimir (Lifshitz) pressure on top of water and ice layers is calculated using Eq. (8) and Eq. (13), and is displayed in Fig. 5] (i) and (ii), respectively. Each panel shows in colour scale the excess pressure as a function of the ice thickness $(a)$, in $x$-axis, and water thickness $(b)$, in $y$-axis, being reddish 


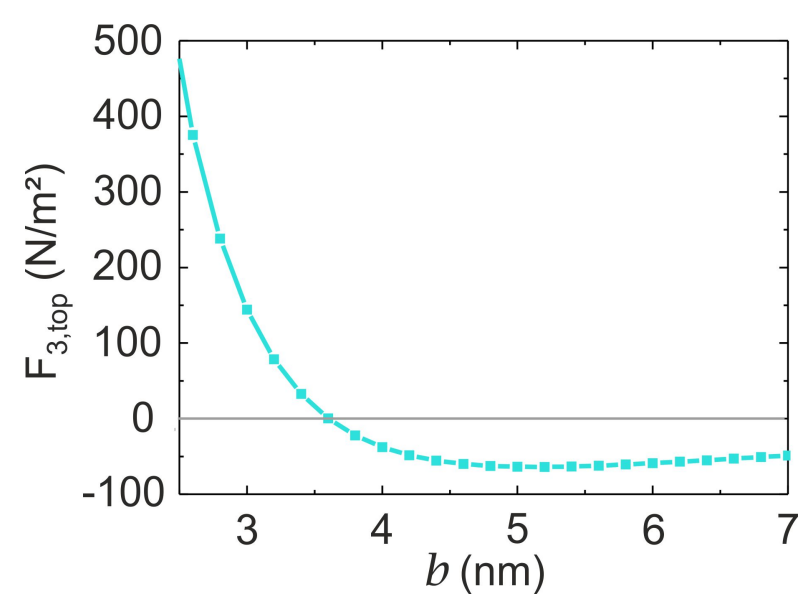

Figure 3: Casimir (Lifshitz) surface pressure $(T=273.16 \mathrm{~K})$ on top of the water layer as a function of water layer thickness $b$, in the limiting case of infinite ice thickness $(a \rightarrow \infty)$ in the 4-layer system.

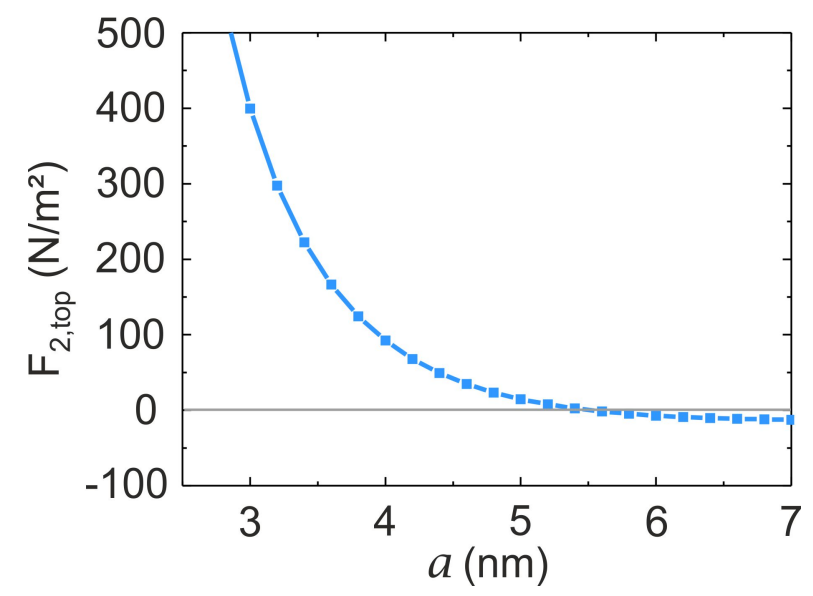

Figure 4: Casimir (Lifshitz) surface pressure $(T=273.16 \mathrm{~K})$ on top of the ice layer as a function of ice layer thickness $a$, in the limiting case of infinite water thickness $(b \rightarrow \infty)$ in the 4-layer system.

colours a positive pressure and blueish colours a negative one over the corresponding interface. White colour indicates regions where the pressure tends to zero. Specifically, since the magnitude of the calculated forces is quite different, a logarithmic scale representation is employed and so, zero force is found in the white regions, which according to the colour scale resolution correspond to pressure values below $10^{-5} \mathrm{~N} / \mathrm{m}^{2}$.

$F_{3, \text { top }}$ and $F_{2, \text { top }}$ show a number of similar features in Fig. 5. Particularly, at small $a$ and $b$ values the force is large and positive, implying a propensity for the water and ice films to grow. At intermediate distances, however, the plots show large blue coloured ponds which correspond to a tendency of the water and ice films to recede. The behaviour resulting from this situation is very interesting. In order to simplify the situation, assume that the system is found exactly at the triple point. According to Eq. [19], mechanical equilibrium corresponds to lines of zero excess force that can be traced easily as the white border between yellowish and blueish regions. For the ice layer, whose growth is dictated by $F_{2 \text {,top }}$, there exist two families of solutions obeying the condition $F_{2 \text {, top }}=0$, corresponding to close to horizontal and vertical isolines along the plot. The former is an ice growth mode, where $a$ can grow boundlessly at $b$ roughly constant and equal to $16 \sim \mathrm{nm}$ (as is usual for ice premelting). The other is a water growth mode, where the ice thickness remains almost constant at $\sim 6 \mathrm{~nm}$, while the water layer grows boundless (as is the case for surface

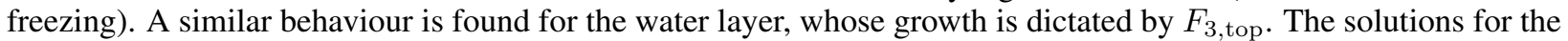
equilibrium condition of the water layer at the triple point is $F_{3 \text {,top }}=0$, and again we find two families of solutions corresponding to a water growth mode and an ice growth mode. In the former case, solutions of $F_{3 \text {,top }}=0$ exist where $b$ grows infinitely while $a$ remains constant at about $12 \mathrm{~nm}$. In the latter case, corresponding to the horizontal line in the plot, the ice layer can diverge while $b$ remains almost constant at less than $4 \mathrm{~nm}$ in thickness (corresponding to the Elbaum and Schick limit). 

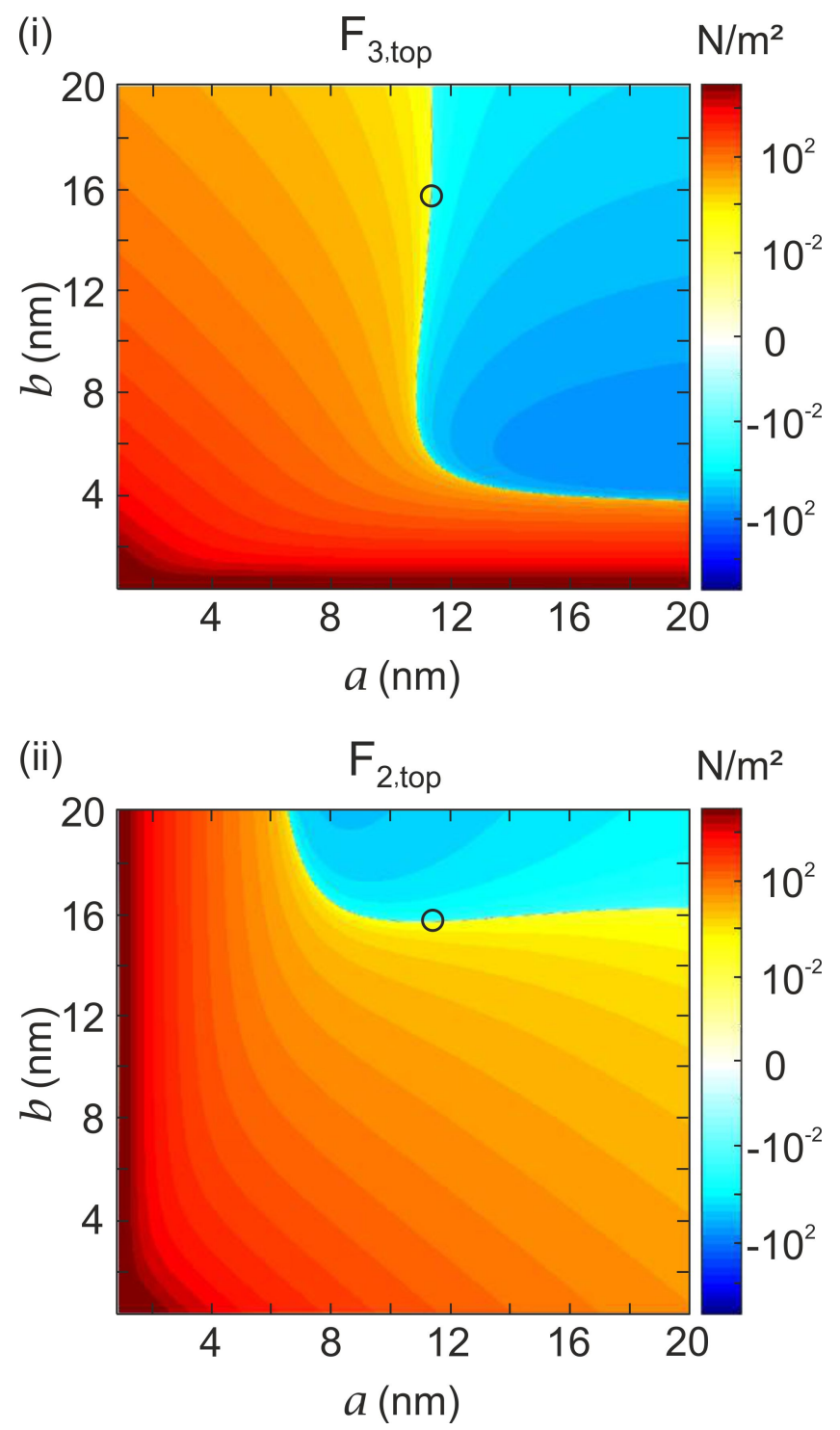

Figure 5: Casimir (Lifshitz) surface pressure $(T=273.16 \mathrm{~K})$ on top of (i) water and (ii) ice layers as a function of ice thickness, $a,(x$-axis) and water thickness, $b$, ( $y$-axis) in the 4-layer system. Positive and negative values mean upwards and downwards pressure, respectively, at the corresponding interface in Fig. 1. In the limit of large $a$ values, in panel (i), the Elbaum-Schick result is recovered, while for large $b$ thicknesses, in panel (ii), we recover our earlier result for solid-ice-water. Black circles stand for the equilibrium point $(12,16) \mathrm{nm}$.

This situation is very interesting and suggestive of the existence of both surface melting and surface freezing on quartz depending on the thermodynamic conditions. In practice, however, the exact equilibrium condition at the triple point is attained when both $F_{2 \text {,top }}=0$ and $F_{3 \text {,top }}=0$ are obeyed simultaneously. From the figures, an intersection of these solutions exists for a thick adsorbed condensate of ice and water with $a \approx 12 \mathrm{~nm}$ and $b \approx 16 \mathrm{~nm}$, which corresponds to an unstable equilibrium (see below). Additional solutions exists for either $a$ or $b$ at infinity, which correspond to solutions at either side of the melting line.

Exploring three-phase coexistence on quartz at arbitrary conditions is difficult, but we can obtain a feeling of the phase behaviour by subtracting $F_{3 \text {,top }}$ from $F_{2 \text {,top }}$, which, according to Eq. (19), yields $F_{2-3}=p_{3}-p_{2}$ as the condition for mechanical equilibrium. $F_{2-3}$ is calculated with Eq. (7) using results from Fig. 5(i) and (ii). Figure 6 shows $F_{2-3}$ as a function of $a$ and $b$. Again, reddish color scale represents positive pressure and blueish, negative pressure, meaning in this case that, in the reddish region $F_{2 \text {,top }}>F_{3 \text {,top }}$, while the opposite occurs in blueish regions, i.e., $F_{2, \text { top }}<F_{3, \text { top }}$. 


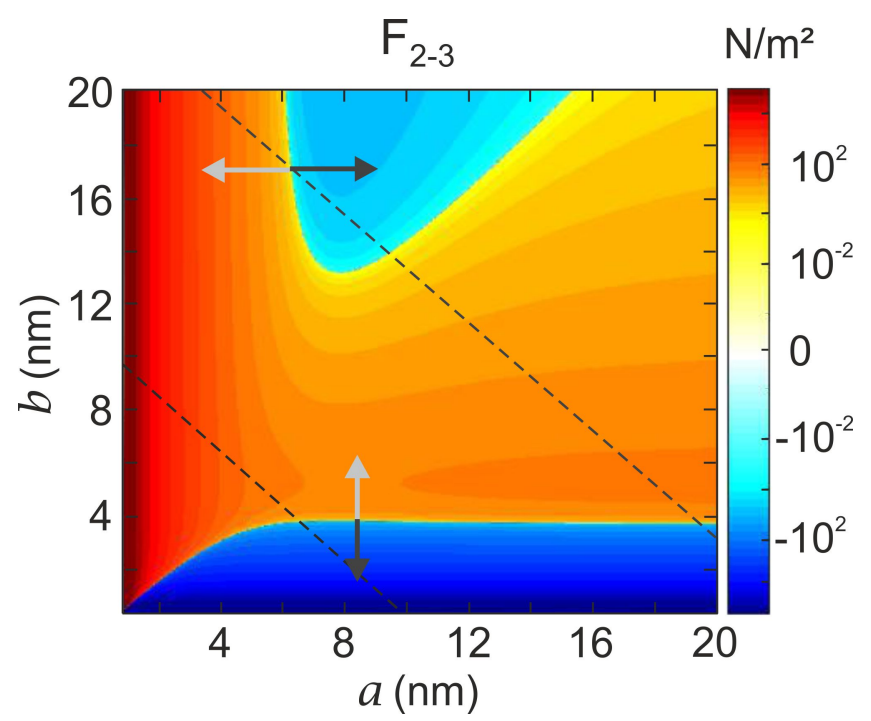

Figure 6: Contour-plot of the Casimir (Lifshitz) pressure $(T=273.16 \mathrm{~K})$ at the ice-water interface as a function of $a$ and $b$. Positive and negative values indicate, $F_{2 \text {,top }}>F_{3 \text {,top }}$ and $F_{2 \text {,top }}<F_{3 \text {,top }}$, correspondingly. The bottom dashed line stands for $a+b=10 \mathrm{~nm}$ and the top dashed line for $a+b=23 \mathrm{~nm}$. Light and dark gray arrows indicate the modes displacement for $T>T_{m}$ and $T<T_{m}$, respectively.

In white regions, separating blueish and reddish regions, pressures are balanced, i.e. $F_{2 \text {,top }}=F_{3, \text { top }}$, and the system is at equilibrium leading to ice-water coexistence.

The figure confirms the scenario suggested from the inspection of $F_{2 \text {,top }}=0$ and $F_{3 \text {,top }}=0$. The system exhibits both a premelted ice growth mode into the Elbaum-Schick limit, corresponding to solutions where the ice layer diverges (with $a \rightarrow \infty$ ) at constant water thickness, $b$; and a surface freezing water growth mode, corresponding to the divergence of the premelting film $(b \rightarrow \infty)$ above a frozen ice layer of constant thickness, $a$. The solutions are separated by a saddle point at ca. $a=8 \mathrm{~nm}$ and $b=6 \mathrm{~nm}$, with a height of $F_{2-3}$ in the hundreds of N/m ${ }^{2}$. Exactly at the triple point, and quite generally, when $p_{2}=p_{3}$, i.e., along the melting line, any of these two solutions is mechanically stable, so the expected outcome of an experiment could largely depend on the initial conditions.

Imagine first a quartz rock that is wet by water at temperatures significantly above the triple point (such that no ice is present). If this rock is suddenly undercooled well below $0^{\circ} \mathrm{C}$, it is suddenly found in a region with $a=0$ and large $b$ values. The system will then grow an ice film until it crosses the line of vertical solutions, and will therefore stabilise a water wet state with a finite amount of surface freezing. On the other hand, if a dry rock is cooled below $0^{\circ} \mathrm{C}$, then initially both $a$ and $b$ are zero, and the likely outcome will be that a thick ice film grows on the rock while water forms a thin premelting film on ice. However, notice that this outcome will depend on the kinetics of water adsorption and ice growth. If the time for adsorbed water to freeze is very large, it could occur that a thick water layer adsorbs well before ice nucleates, and then the system would also move to the line of water wet solutions.

What happens away from the triple point? In fact, solutions of $F_{2-3}=0$ correspond to the condition $p_{3}=p_{2}$, and are valid for all points along the melting line, where the pressure of water and ice are equal. Away from this line, the condition of equilibrium reads $F_{2-3}=p_{3}-p_{2}$. To gauge the relevant energy scale, note that $p_{3}-p_{2}=\Delta H \rho_{w} \frac{T-T_{m}}{T_{m}}$, with $\Delta H$, the enthalpy of fusion, $\rho_{w}$ the water density, and $T_{m}$ the melting temperature. This provides $p_{3}-p_{2} \approx$ $10^{6}\left(T-T_{m}\right) \mathrm{N} / \mathrm{m}^{2}$, corresponding to a rise of 10 atmospheres for every Kelvin away from the melting line. This is a huge amount for large films, but is in the scale of the Casimir (Lifshitz) forces expected for subnanometer thicknesses, since then the forces increase steeply with the inverse cube of the film thickness. At a temperature above the melting line $T>T_{m}$, then $p_{3}>p_{2}$, and those solutions with positive $F_{2-3}$ will be selected. For the water growth mode (the white vertical line in Fig. 6), this moves the equilibrium condition towards smaller values of $a$, as expected, since the ice phase becomes unfavourable. A large temperature difference is needed to decrease $a$, since the Casimir (Lifshitz) force for small thicknesses increases steeply. For the ice growth mode (the horizontal line in Fig 6), on the contrary, moving into $F_{2-3}>0$ leaves the ice thickness unchanged, but increases $b$, as water is now favoured over ice. Eventually, if $p_{3}-p_{2}$ becomes large enough, then the solutions move away from the stability basin of the ice-dominant region, across the saddle point into the water growth mode. Notice from the scale this barrier is only in the hundreds of $\mathrm{N} / \mathrm{m}^{2}$, so that merely an increase of $10^{-4} \mathrm{~K}$ is sufficient to move from the ice growth to the water growth mode. At temperatures 
below the melting line, $T<T_{m}$, on the other hand, $p_{2}>p_{3}$, and those solutions with negative $F_{2-3}$ will be selected. For the water growth mode, moving towards $F_{2-3}<0$ drives the system into the blue region, which is achieved at almost constant water thickness, by increasing $a$, that is, by growing the surface freezing layer. For the ice growth mode, moving into the blue region on the contrary is achieved by keeping $a$ almost constant, and decreasing the premelting layer thickness. This is precisely what happens by cooling ice along the sublimation line, where the premelting layer thickness is known to decrease from several nanometers thickness at the triple point to well below a nanometer at $-10 \mathrm{C}$.

Notice that this discussion has assumed an open system from the outset, i.e., we have assumed there is an infinite vapour reservoir to feed the growth of either ice or water, as in the atmosphere. It is now interesting to inspect how the behaviour is determined in a situation where the thickness of ice plus water is limited, i.e. $a+b$ (roughly) constant, as in a closed cavity or a slit pore formed within the rock. Dashed black lines in Fig. 6 represents cavity thicknesses of $10 \mathrm{~nm}$ (bottom line) and $23 \mathrm{~nm}$ (top line). In the first case, the line crosses a white region at the point ( $a \approx 6 \mathrm{~nm}$, $b \approx 4 \mathrm{~nm}$ ) meaning that the system is at equilibrium at this layer thickness combination. Notice that at this point, a perturbation decreasing $a$ moves the system to the region $F_{2 \text {,top }}>F_{3 \text {,top }}$, so that the ice layer grows and the perturbation is restored. i.e., this solution does indeed correspond to a state of mechanical equilibrium. This result lends support to the existence of premelting in nanoscale cavities. [7] For an amount of condensed water of $\approx 20 \mathrm{~nm}$, the system reaches the equilibrium at three different points as shown for the top dashed line with $a+b=23 \mathrm{~nm}$. Here, both water wet and ice wet solutions are possible. Also note that an intermediate solution with $a=5 \mathrm{~nm}$ and $b=14 \mathrm{~nm}$ exists, but this is an unstable state. If $a$ here is increased as a result of a perturbation, the system is moved into where $F_{2, \text { top }}>F_{3, \text { top }}$ and the perturbation is amplified.

\section{Conclusions}

An improved theory for Casimir (Lifshitz) forces in four layer systems has been derived within the stress tensor approach. To explore the new stress tensor theory we use in the present work the commonly accepted models for ice and water prepared by Elbaum and Schick. [1] As noted the theory presented in our work enables studies in multilayered systems where more than one film thickness can be varied. However, it should be stressed that very small changes in dielectric functions for water or ice (originating for instance from impurities, [4, 26] presence of salt ions, [4, 16, 5] or from modelling based on a different set of optical data [99]) could influence the results, such as where premelting and ice formation near interfaces will occur. This sensitivity comes from the closeness of dielectric functions for ice and water as was pointed out many years ago by Elbaum and Schick. [1]

In contrast to previous works on multilayered Casimir [92] (Lifshitz [53]) force theory, we considered the electromagnetic stress tensor in the reference and in its neighbouring layer. This consideration allows the reduction of the four layer system to both corresponding three layer systems. In particular, by setting $a \rightarrow \infty$ the premelting of ice case [1] can be derived. But also the ice formation at some solid-water interfaces (e.g. silica [97] and some gas hydrates [26]) can be extracted by setting $b \rightarrow \infty$. Beyond this reproduction of previous results, the different stress tensors strongly influence the results for small layer thicknesses of the neighbouring layer. This could for instance influence the escape of greenhouse gas molecules from melting ice. [100, 101]

It has been speculated that a liquid-vapour surface could help nucleate ice. [102] Here, and in previous work [8], we show that ice has a propensity to grow up to about $3.6 \mathrm{~nm}$ close to the liquid-vapour surface, since this slightly stabilises the system with respect to the formation of two distant ice-liquid and liquid-vapour interfaces. Application of our theory enabled us to demonstrate the thickness dependence of the premelting of an ice layer attached to a quartz rock surface. For very thick ice layers we recover as already discussed the results from the corresponding three layer ice-water-vapour system from the work by Elbaum and Schick. [1] As in their original work, a short-range repulsive interaction goes over to a long-range attraction. A thin film of water on ice is thus supported by an energy minimum in the Casimir (Lifshitz) energy. However, another family of solutions also exists where a thick water film can grow on top of a thin ice layer adsorbed on the rock, as in surface freezing. For a system with thin films of both ice and water, growing on a rock surface, we have seen a much more complex and interesting scenario with more than one possible equilibrium for the system depending on initial conditions.

\section{Conflicts of interest}

There are no conflicts of interest to declare. 


\section{Acknowledgements}

Especially, we would like to thank Stefan Scheel and Kimball A. Milton for fruitful discussions. We thank Barry W. Ninham for careful readings of different versions of this manuscript and providing useful feedback. We acknowledge support from the Research Council of Norway (Project 250346) and the Spanish Ministry of Science, Innovation and Universities for funding under grant EXPLORA FIS2017-91018-EXP and FIS2017-89361-C3-2-P. S.C.-P. acknowledges for funding through a Juan de la Cierva Incorporación under grant IJCI-2016-28549. We gratefully acknowledge support by the German Research Council (grant BU 1803/6-1, S.Y.B. and J.F., BU 1803/3-1, S.Y.B. and F.S.). V.E. is grateful to "la Caixa" Foundation (ID 100010434) for its support through a fellowship with code: LCF/BQ/ES15/10360025.

\section{References}

[1] M. Elbaum and M. Schick, Phys. Rev. Lett., 1991, 66, 1713-1716.

[2] M. Elbaum, S. Lipson and J. Dash, Journal of Crystal Growth, 1993, 129, 491 - 505.

[3] L. A. Wilen, J. S. Wettlaufer, M. Elbaum and M. Schick, Phys. Rev. B, 1995, 52, 12426-12433.

[4] J. S. Wettlaufer, Phys. Rev. Lett., 1999, 82, 2516-2519.

[5] P. Thiyam, J. Fiedler, S. Y. Buhmann, C. Persson, I. Brevik, M. Boström and D. F. Parsons, The Journal of Physical Chemistry C, 2018, 122, 15311-15317.

[6] J. S. Wettlaufer, Philosophical Transactions of the Royal Society A: Mathematical, Physical and Engineering Sciences, 2019, 377, 20180261.

[7] H. Li, M. Bier, J. Mars, H. Weiss, A.-C. Dippel, O. Gutowski, V. Honkimäki and M. Mezger, Phys. Chem. Chem. Phys., 2019, 21, 3734-3741.

[8] P. Parashar, K. V. Shajesh, K. A. Milton, D. F. Parsons, I. Brevik and M. Boström, Phys. Rev. Research, 2019, 1, 033210.

[9] T. Mitsui and K. Aoki, Phys. Rev. E, 2019, 99, 010801.

[10] R. Bar-Ziv and S. A. Safran, Langmuir, 1993, 9, 2786-2788.

[11] M. Boström, O. I. Malyi, P. Thiyam, K. Berland, I. Brevik, C. Persson and D. F. Parsons, EPL (Europhysics Letters), 2016, 115, 13001.

[12] B. W. Ninham and V. Yaminsky, Langmuir, 1997, 13, 2097-2108.

[13] M. Boström, D. R. M. Williams and B. W. Ninham, Phys. Rev. Lett., 2001, 87, 168103.

[14] B. W. Ninham and P. Lo Nostro, Molecular Forces and Self Assembly in Colloid, Nano Sciences and Biology, Cambridge University Press, Cambridge, 2010.

[15] D. F. Parsons, M. Boström, P. L. Nostro and B. W. Ninham, Phys. Chem. Chem. Phys., 2011, 13, $12352-12367$.

[16] P. Thiyam, E. R. A. Lima, O. I. Malyi, D. F. Parsons, S. Y. Buhmann, C. Persson and M. Boström, EPL (Europhysics Letters), 2016, 113, 43002.

[17] M. Baker and J. Dash, Journal of Crystal Growth, 1989, 97, 770 - 776.

[18] J. G. Dash and J. S. Wettlaufer, Canadian Journal of Physics, 2003, 81, 201-207.

[19] S. C. Sherwood, V. T. J. Phillips and J. S. Wettlaufer, Geophysical Research Letters, 2006, 33, L05804.

[20] L. A. Wilen and J. G. Dash, Phys. Rev. Lett., 1995, 74, 5076-5079.

[21] J. G. Dash, H. Fu and J. S. Wettlaufer, Reports on Progress in Physics, 1995, 58, 115.

[22] J. G. Dash, A. W. Rempel and J. S. Wettlaufer, Rev. Mod. Phys., 2006, 78, 695-741.

[23] D. T. Möhlmann, Icarus, 2004, 168, 318 - 323.

[24] D. T. Möhlmann, Icarus, 2008, 195, 131 - 139.

[25] H. Hansen-Goos, E. S. Thomson and J. Wettlaufer, Planetary and Space Science, 2014, 98,169 - 181.

[26] M. Boström, R. W. Corkery, E. R. A. Lima, O. I. Malyi, S. Y. Buhmann, C. Persson, I. Brevik, D. F. Parsons and J. Fiedler, ACS Earth and Space Chemistry, 2019, 3, 1014-1022.

[27] K.-i. Murata, H. Asakawa, K. Nagashima, Y. Furukawa and G. Sazaki, Proc. Nat. Acad. Sci., 2016, 113, E6741-E6748.

[28] B. Slater and A. Michaelides, Nat. Rev. Chem, 2019, 3, 172-188. 
A PREPRINT - FEBRUARY 12, 2021

[29] M. M. Conde, C. Vega and A. Patrykiejew, J. Chem. Phys., 2008, 129, 014702.

[30] H. Bluhm, D. F. Ogletree, C. S. Fadley, Z. Hussain and M. Salmeron, J. Phys.: Condens. Matter, 2002, 14, L227-L233.

[31] J. Gelman Constantin, M. M. Gianetti, M. P. Longinotti and H. R. Corti, Atmospheric Chemistry and Physics, 2018, 18, 14965-14978.

[32] N. Kallay, A. Čop, E. Chibowski and L. Holysz, Journal of Colloid and Interface Science, $2003,259,89$ - 96.

[33] A. D. McLachlan, Proceedings of the Royal Society of London A: Mathematical, Physical and Engineering Sciences, 1963, 271, 387-401.

[34] H. B. G. Casimir, K. Ned. Akad. Wet., 1948, 51, 793.

[35] H. B. G. Casimir and D. Polder, Phys. Rev., 1948, 73, 360-372.

[36] J. Perina, Coherence and Statistics of Photons and Atoms, Wiley, New York, 2001.

[37] S. Y. Buhmann, Dispersion Forces I: Macroscopic quantum electrodynamics and ground-state Casimir, CasimirPolder and van der Waals forces, Springer, Heidelberg, 2012.

[38] D. Tabor, Journal of Colloid and Interface Science, 1980, 75, 240 - 245.

[39] Brittish Museum, Cuneiform Texts from Babylonian Tablets in Brittish Museum, Vol. 5, Plate IV, 22446, London, 1898.

[40] C. W. Ceram, Goods, Graves \& Scholars. The story of archeology, second revised edition, Vintage books, New York, 1986.

[41] T. Young, Phil. Trans. Royal Soc. London, 1805, 95, 65.

[42] J. G. Dash, Contemporary Physics, 1989, 30, 89-100.

[43] B. V. Derjaguin, I. I. Abrikosova and E. M. Lifshitz, Q. Rev. Chem. Soc., 1956, 10, 295-329.

[44] P. Lebedew, Annalen der Physik, 1894, 288, 621-640.

[45] F. London, Z. Phys. Chem. B, 1930, 11, 222.

[46] E. M. Lifshitz, Soviet Physics JETP 2, Part 1, 1956, 2, 73-83.

[47] I. E. Dzyaloshinskii, E. M. Lifshitz and L. P. Pitaevskii, Advances in Physics, 1961, 10, 165-209.

[48] B. W. Ninham, V. A. Parsegian and G. H. Weiss, J. Stat. Phys., 1970, 2, 323-328.

[49] B. W. Ninham and V. A. Parsegian, The Journal of Chemical Physics, 1970, 52, 4578-4587.

[50] B. W. Ninham and V. A. Parsegian, The Journal of Chemical Physics, 1970, 53, 3398-3402.

[51] P. Richmond and B. W. Ninham, Solid State Communications, 1971, 9, 1045 - 1047.

[52] V. A. Parsegian and B. W. Ninham, J. Theor. Biol., 1973, 38, 101 - 109.

[53] P. Richmond, B. W. Ninham and R. Ottewill, Journal of Colloid and Interface Science, 1973, 45, 69 - 80.

[54] P. Richmond and B. W. Ninham, Journal of Physics C: Solid State Physics, 1971, 4, 1988-1993.

[55] J. Mahanty and B. W. Ninham, Dispersion Forces, Academic Press, London, 1976.

[56] S. J. Rahi, M. Kardar and T. Emig, Phys. Rev. Lett., 2010, 105, 070404.

[57] B. W. Ninham and V. Parsegian, Biophysical Journal, 1970, 10, 646 - 663.

[58] P. J. van Zwol, G. Palasantzas and J. T. M. De Hosson, Phys. Rev. B, 2009, 79, 195428.

[59] P. J. van Zwol and G. Palasantzas, Phys. Rev. A, 2010, 81, 062502.

[60] V. A. Parsegian, Van der Waals forces: A handbook for biologists, chemists, engineers, and physicists, Cambridge University Press, New York, 2006.

[61] S. Y. Buhmann, Dispersion Forces II: Many-Body Effects, Excited Atoms, Finite Temperature and Quantum Friction, Springer, Heidelberg, 2012.

[62] B. E. Sernelius, Fundamentals of van der Waals and Casimir Interactions, Springer International Publishing, 2018.

[63] V. Esteso, S. Carretero-Palacios and H. Miguez, The Journal of Physical Chemistry C, 2015, 119, 5663-5670.

[64] V. Esteso, S. Carretero-Palacios and H. Miguez, Journal of Applied Physics, 2016, 119, 144301.

[65] V. Esteso, S. Carretero-Palacios and H. Miguez, The Journal of Physical Chemistry Letters, 2019, 10, 5856-5860. 
[66] C. H. Anderson and E. S. Sabisky, Phys. Rev. Lett., 1970, 24, 1049-1052.

[67] P. Richmond and B. Ninham, J. Low. Temp. Phys, 1971, 5, 177 - 189.

[68] E. S. Sabisky and C. H. Anderson, Phys. Rev. A, 1973, 7, 790-806.

[69] H. T. Dung, L. Knöll and D.-G. Welsch, Phys. Rev. A, 1998, 57, 3931-3942.

[70] F. Hauxwell and R. Ottewill, Journal of Colloid and Interface Science, 1970, 34, 473 - 479.

[71] A. Milling, P. Mulvaney and I. Larson, J. Colloid Interface Sci., 1996, 180, 460.

[72] S. Lee and W. M. Sigmund, J. Colloid Interface Sci., 2001, 243, 365.

[73] A. A. Feiler, L. Bergström and M. W. Rutland, Langmuir, 2008, 24, 2274.

[74] J. N. Munday, F. Capasso and V. A. Parsegian, Nature, 2009, 457, 170.

[75] R. Zhao, L. Li, S. Yang, W. Bao, Y. Xia, P. Ashby, Y. Wang and X. Zhang, Science, 2019, 364, 984-987.

[76] M. Bordag, G. L. Klimchitskaya, U. Mohideen and V. M. Mostepanenko, Advances in the Casimir Effect, Oxford Science Publications, Oxford, 2009.

[77] K. A. Milton, The Casimir effect: Physical manifestations of zero-point energy, World Scientific, River Edge, USA, 2001.

[78] V. A. Parsegian, Van der Waals Forces, Cambridge University Press, New York, 2006, p. 398.

[79] B. W. Ninham and P. Lo Nostro, Molecular Forces and Self Assembly In Colloid, Nano Sciences and Biology, Cambridge University Press, Cambridge, 2010.

[80] W. M. R. Simpson and U. Leonhardt, Forces of the Quantum Vacuum, World Scientific, 2015.

[81] Handbook of Physical Quantities, ed. I. S. Grigoriev and E. Z. Meilikhov, CRC Press, New York, 1997.

[82] R. Evans, J. R. Henderson, D. C. Hoyle, A. O. Parry and Z. Sabeur, Mol. Phys., 1993, 80, 755-775.

[83] J. R. Henderson, Phys. Rev. E, 1994, 50, 4836-4846.

[84] J. Benet, J. G. Palanco, E. Sanz and L. G. MacDowell, J. Phys. Chem. C, 2014, 118, 22079-22089.

[85] J. Benet, P. Llombart, E. Sanz and L. G. MacDowell, Mol. Phys., 2019, 117, 2846-2864.

[86] J. Benet, P. Llombart, E. Sanz and L. G. MacDowell, Phys. Rev. Lett., 2016, 117, 096101.

[87] I. Pickering, M. Paleico, Y. A. P. Sirkin, D. A. Scherlis and M. H. Factorovich, J. Phys. Chem. B, 2018, 122, 4880-4890.

[88] Y. Qiu and V. Molinero, J. Phys. Chem. Lett., 2018, 9, 5179-5182.

[89] M. Alheshibri, J. Qian, M. Jehannin and V. S. J. Craig, Langmuir, 2016, 32, 11086-11100.

[90] B. W. Ninham, R. M. Pashley and P. L. Nostro, Current Opinion in Colloid \& Interface Science, 2017, 27, 25 32.

[91] M. Boström and B. E. Sernelius, Physica A, 2004, 339, 53.

[92] C. Raabe, L. Knöll and D.-G. Welsch, Phys. Rev. A, 2003, 68, 033810.

[93] S. A. Ellingsen, Journal of Physics A: Mathematical and Theoretical, 2007, 40, 1951-1961.

[94] F. A. Burger, J. Fiedler and S. Y. Buhmann, EPL, 2018, 121, 24004.

[95] P. A. Maia Neto, F. S. S. Rosa, L. B. Pires, A. B. Moraes, A. Canaguier-Durand, R. Guérout, A. Lambrecht and S. Reynaud, The European Physical Journal D, 2019, 73, 178.

[96] D. Sibley, P. Llombart, E. G. Noya, A. Archer and L. G. MacDowell, arXiv:2003.04252, 2020, -.

[97] M. Boström, O. I. Malyi, P. Parashar, K. V. Shajesh, P. Thiyam, K. A. Milton, C. Persson, D. F. Parsons and I. Brevik, Phys. Rev. B, 2017, 95, 155422.

[98] M. Elbaum and M. Schick, J. Phys. I France, 1991, 1, 1665-1668.

[99] J. Fiedler, M. Boström, C. Persson, I. H. Brevik, R. W. Corkery, S. Y. Buhmann and D. F. Parsons, J. Phys. Chem. $B, 2020$.

[100] P. Llombart, R. M. Bergua, E. G. Noya and L. G. MacDowell, Phys. Chem. Chem. Phys., 2019, 21, 19594-19611.

[101] J. Fiedler, D. F. Parsons, F. A. Burger, P. Thiyam, M. Walter, I. Brevik, C. Persson, S. Y. Buhmann and M. Boström, Phys. Chem. Chem. Phys., 2019, 21, 21296-21304.

[102] A. Haji-Akbari and P. G. Debenedetti, Proc. Nat. Acad. Sci., 2017, 114, 3316-3321. 